\title{
Moving in synchrony with an avatar - presenting a novel and unbiased body sway synchronization paradigm
}

\author{
Clara Scheer $^{1}$ (D) - Lisa Horn ${ }^{2} \cdot$ Petra Jansen $^{1}$ \\ Accepted: 10 March 2021 / Published online: 16 April 2021 \\ (C) The Author(s) 2021
}

\begin{abstract}
Moving in synchrony with one another is a fundamental mechanism that maintains human social bonds. Yet, not all individuals are equally likely to coordinate their behaviors with others. The degree of interpersonal coordination is greatly influenced by preexisting characteristics of the interacting partners, like the cultural homogeneity of a group, shared goals, and the likability of the other person. Considering that most research questions necessitate an experimental set-up without such uncontrolled biases, we created a novel, unbiased paradigm: a human-avatar body sway synchronization paradigm. Participants' body sway was measured by a force plate while being exposed to a medio-laterally moving avatar. Forty-nine participants were tested in a social condition (motionless vs. moving avatar) and a non-social control condition (motionless vs. moving column). The results revealed that participants increased their body sway on their medio-lateral axis while the avatar was moving. The participants did not increase their body sway in the non-social control condition, indicating that the participant's movement was not simply caused by a basal motion perception process. The current study builds a methodological fundament that can help to reduce biases due to pre-existing rapport between interaction partners and serves as a valuable experimental paradigm for future synchrony studies.
\end{abstract}

Keywords Interpersonal synchrony $\cdot$ Synchronization paradigm $\cdot$ Avatar $\cdot$ Force plate $\cdot$ Body sway

\section{Introduction}

Coordinating gestures and movements with one another has been proposed as one of the fundamental mechanisms underlying the establishment and maintenance of human social bonds (Bernieri \& Rosenthal, 1991). The existing literature points towards a bidirectional, conducive relationship between interpersonal coordination and affiliation: not only does interpersonal coordination increase liking between the interaction partners (Valdesolo \& DeSteno, 2011), traits that evoke likeability, rapport, and similarity also increase the degree of interpersonal coordination (e.g., McIntosh, 2006; Miles et al., 2010; Néda et al., 2000). Considering that most research questions necessitate an experimental set-up without uncontrolled biases resulting from pre-existing differences in rapport

Clara Scheer

clara.scheer@psk.uni-regensburg.de

1 Faculty of Human Sciences, University of Regensburg, Universitätsstraße 31, 93053 Regensburg, Germany

2 Department of Behavioral and Cognitive Biology, University of Vienna, Althanstraße 14, 1090 Vienna, Austria between the interaction partners, the need for novel and unbiased interpersonal coordination paradigms becomes clear. Therefore, this study will present an innovative experimental set-up that aims to eliminate any pre-existing biases and that can be used as potential basis for future research into interpersonal coordination.

\section{Prior Literature}

Interpersonal coordination is generally understood as nonrandom, patterned or synchronized behaviors that overlap in time and form between two or more people (Bernieri et al., 1988, p. 403). It can take the form of interpersonal movement synchrony, for which the concurrent timing of the movement is critical, and behavior mimicry, where matching behaviors occur sequentially (Chartrand \& Lakin, 2013). From a theoretically point of view, interpersonal synchrony has been argued to be a self-organizing process like the dynamics of a cardiac pacemaker (de Bruin et al., 1983). Movements of individuals are coupled and become mutually synchronous over time, either in-phase (i.e. occurring at the same time) or anti-phase (i.e. occurring in alternation) (for an review see: Ravignani, 2015). These coordination dynamics have been explored both 
mathematically (Haken et al., 1985) and empirically (for an overview see: Schmidt \& Richardson, 2008). The theory behind mimicry has been explained through the perceptionbehavior link, where representations of an action are automatically followed by a behavior (Chartrand \& van Baaren, 2009). Above all, interpersonal coordination is a fundamental part of many cultural traditions, like music-making, dancing, or singing (Hagen \& Bryant, 2003). But interpersonal synchrony and behavior mimicry also occur unintentionally, both in dyadic interactions (e.g., Chartrand et al., 2005) and in large groups of people (e.g., Néda et al., 2000). As an integral part of human social interactions, interpersonal synchrony and behavior mimicry already play an important role in early exchanges between infants and their caregivers (Condon \& Sander, 1974). Adults have been found to synchronize their movements in a variety of contexts; e.g. walking (Wiltermuth \& Heath, 2009), music making (Chang et al., 2019), rocking in rocking chairs (Richardson et al., 2007)) and sequentially match others' gestures, postures and motor movements (Chartrand \& Lakin, 2013). Observing a point-light display of a balancing gymnast, for example, can induce corresponding body sway in participants (Tia et al., 2011).

Most research focuses on the effects of interpersonal coordination on the interactants, which are usually positive. The most commonly agreed findings are: an increase of liking and the feeling of unity and similarity between interacting partners, which can subsequently lead to an increase in costly helping behavior and altruism (Valdesolo \& DeSteno, 2011). Experiencing interpersonal coordination also leads to enhanced social cognition skills and prosocial behavior (Valdesolo \& DeSteno, 2011; Vicaria \& Dickens, 2016).

At the same time, researchers have long been intrigued by questions about which factors cause individuals to coordinate themselves with another person. Not all individuals were found to be equally likely to coordinate their behavior and even inconspicuous traits of an individual can influence the degree of synchronization. For example, groups with a strong cultural homogeneity (Néda et al., 2000) and participants with shared goals (Reddish et al., 2013) are better at synchronizing their behavior. Moreover, pro-socially orientated people coordinate their movements with others better than individuals with a pro-selforientation (Lumsden et al., 2012) and individuals with a greater disposition to empathic understanding show stronger behavior mimicry (Chartrand \& Bargh, 1999; Sonnby-Borgström et al., 2003). When investigating the effect of a participant's mood on interpersonal coordination, results have been mixed so far: participants in a positive mood have been found to mimic a confederate significantly more often than participants in a negative mood (e.g., Van Baaren et al., 2006), although interpersonal movement synchrony was not found to increase after induced positive or negative mood (Fujiwara \& Daibo, 2018).

Additionally, also the traits and/or behavior of the interaction partner can influence the degree of interpersonal coordination. Miles et al. (2010) found that participants were less likely to synchronize their movements with a confederate who arrived late for the experiment compared to one who arrived on time (Miles et al., 2010). Similarly, likeable confederates are mimicked more than unlikeable confederates (McIntosh, 2006) and in-group members are mimicked more than out-group members (Mondillon et al., 2007). Even sharing the same name was found to lead to more coordinated behavior (Guéguen \& Martin, 2009). These findings demonstrate the potential impact of pre-existing traits of a confederate and the possible influence of a partner's behavior on subsequent interpersonal coordination. Although interpersonal coordination has so far mostly been investigated from the point of view of a truly social interaction between individuals, the presented findings also highlight the need for neutral experimental designs that allow minimizing unconscious biases resulting from pre-existing differences in rapport with a real interaction partner.

Due to the fact that interpersonal coordination can be found in multiple aspects of bodily movement, researchers have so far used many different movement tasks to assess the coordination between interaction partners, e.g. clapping (Néda et al., 2000), walking (Van Ulzen et al., 2007), rocking in a chair (Richardson et al., 2007), pen playing (Van Baaren et al., 2006), or face touching (Lakin \& Chartrand, 2003). Another naturalistic and at the same time accurate way to assess body movements (and consequentially interpersonal coordination) in the laboratory is body sway. Natural body sway consists of movements around an individual's center of gravity, mostly on the level of micro movements (Nishiyama et al., 2016), although also larger body movements have been described as body sway (Chang et al., 2020). Body sway can be reliably assessed by using the technology of motion capture (Chang et al., 2019, 2020) or of a force plate (Reynolds \& Osler, 2014). Previous work on coordinated body sway found that synchronous body movement within a musical ensemble was greater when the individual members were told to perform a piece with emotional expression than without this instruction (Chang et al., 2019). Also, romantic interest in dyads is reflected by a non-verbal body sway. It is shown that movements of one partner predicted the movements of the other partner and that a high directional coupling body sway correlates with interest in long-term relationships (Chang et al., 2020). These findings show that natural body sway is a promising candidate behavior for investigating interpersonal coordination.

\section{Present Study}

The present study aims to present a novel coordination paradigm that eliminates pre-existing biases between real interaction partners, namely a human-avatar body sway coordination paradigm. By combining the benefits of measuring natural 
body sway with a neutral looking avatar this experimental paradigm can be used as potential basis for future research. Recent human-avatar coordination studies found that humanavatar interactions were comparable with human-human interactions when participants were told to actively follow an avatar's movements (Meerhoff et al., 2017; Sacheli et al., 2015). In all these studies, movement coordination was explicitly triggered by experimenter instructions. In this study we want to exclude the influence of the experimenter instruction: Do participants spontaneously and without external request synchronize their movements with an avatar's movements? To test this, we exposed participants to the projection of a human-like, gender-neutral, life-sized avatar, which showed body sway on the medio-lateral axis for one minute, preceded and followed by baseline phases without movement. We assessed whether the participants would alter their natural body sway in response to the moving avatar. To control whether synchronization effects were dependent on the avatar's resemblance to a human or whether they could also be elicited by the simple perception of motion, each participant additionally received the experimental treatment with the projection of a similarly sized, neutral object (i.e., a column). Since we wanted to model our set-up as closely as possible to interpersonal coordination, which can happen unintentionally and effortlessly (Chartrand \& Lakin, 2013), we diverted the participants' conscious attention from the projection of the avatar or the column to a secondary task. Additionally, we used questionnaires to assess the participants' dispositional empathy and current mood.

According to our hypotheses, we firstly expected participants to unconsciously coordinate their body sway with the moving avatar (i.e., increase their body sway when the avatar started its medio-lateral movement). Secondly, we predicted that participants would coordinate their movements to a higher degree with the movement of the avatar that resembled a human figure, than with a moving neutral object. Thirdly, we hypothesized that participants with higher empathic abilities would be more likely to coordinate their movements with the moving avatar, as higher empathic abilities were found to increase interpersonal synchrony and behavior mimicry in human-human interactions (Chartrand \& Bargh, 1999; Sonnby-Borgström et al., 2003). Since mood has been found to positively affect mimicry (e.g. Van Baaren et al., 2006), we expected to find a similar effect in our study.

\section{Methods}

\section{Participants}

49 healthy young adults (35 females and 14 males) volunteered and gave written informed consent to participate in the study (mean \pm SD: female's age $20.40 \pm 2.61$; male's age $23.36 \pm 3.23$ ). Six participants had to be excluded due to failure of the technical equipment or experimenter error. ${ }^{1}$ The experimental procedure was approved by the ethical committee of the University of Regensburg and conducted according to the ethical guidelines of Helsinki.

\section{Procedure}

At arrival, the participants were asked to fill out two questionnaires: The Questionnaire of Cognitive and Affective Empathy (QCAE) (Reniers et al., 2011) and a multidimensional mood questionnaire (Mehrdimensionale Befindlichkeits-Fragebogen - MBDF) (Steyer et al., 1994). During the main experimental data collection, participants were standing barefoot on a forceplate (AMTI OR6-7-2000 Series Model Force Platforme, Watertown, MA 02472, USA). The participants' feet were approximately $22 \mathrm{~cm} / 9$ in. apart and at an angle of $30^{\circ}$ from the force plate's middle line. The force plate was positioned $250 \mathrm{~cm} / 98.4 \mathrm{in}$. away from two screens: a large screen $(180 \times 120 \mathrm{~cm} / 70.9 \times 47.2 \mathrm{in}$. $)$ and a small TV screen $(106.7 \mathrm{~cm} / 42$ in. screen diagonal) situated on a table in front of the large screen (Fig. 1). The participants were asked to stand quietly on both feet with their arms hanging at their sides and to pay attention to the pictures on the TV screen, which were selected neutral pictures of the IAPS (International Affective Picture System) (Lang et al., 2008). To assure that the participants attended to the content on the TV screen, they were informed at the beginning of the experiment that they would later be questioned about the content of the pictures displayed there. In a debriefing, participants were asked whether they noticed a change of movement on the large screen after finishing the experiment. Only a very limited number of participants reported noticing a swaying movement on the large screen and none of them correctly deduced the aim of our study, therefore we decided not to investigate this further. While the pictures were presented on the TV screen, on the large screen either the avatar (social condition) or the column, which had the same height and width as the avatar (non-social control condition) were projected. The sequence of the two conditions was counterbalanced across participants. At the beginning of each trial, either the avatar or the column was presented standing completely still (motionless) for $30 \mathrm{~s}$ (baseline phase 1). Thereafter, the projected stimulus started swaying along the medio-lateral axis (i.e. from the left side to the right side and back) for $1 \mathrm{~min}$ (experimental phase). The swaying frequency resembled the natural body sway frequency (i.e. below $1 \mathrm{~Hz}$; Funato et al., 2016). This phase was followed by another non-moving (motionless) phase of $30 \mathrm{~s}$

\footnotetext{
${ }^{1}$ Prior to testing the force plate had to be set to zero line; in four cases this procedure did not work according to the instructions or was omitted by the experimenter. One person asked to withdraw from the study after data collection, and during one trial for unknown technical reasons no data were collected.
} 
Fig. 1 Experimental set-up. 1: participant, 2: avatar during social condition, column during nonsocial control condition, 3: large screen, 4: TV-screen, 5:

forceplate, 6: amplifier, 7: Vicon Nexus recorder

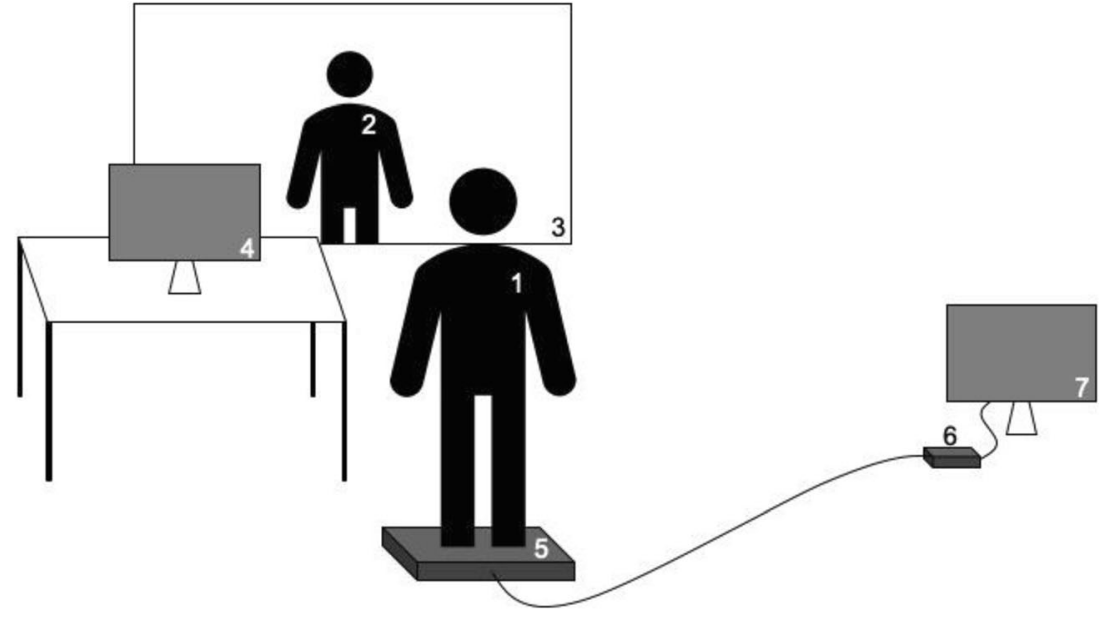

(baseline phase 2). There was a break of $15 \mathrm{~s}$ between the social condition and the non-social control condition during which participants were allowed to move freely.

\section{Measures}

The participants' medio-lateral movements were recorded using a forceplate (AMTI OR6-7-2000 Series Model Force Platforme, Watertown, MA 02472, USA) at $960 \mathrm{~Hz}$; no online filters were applied during the recording of the data.

Both questionnaires, the QCAE (Reniers et al., 2011) and the MBDF (Steyer et al., 1994) were answered before the experiment started. The online questionnaires were generated using SoSci Survey (Leiner, 2019) and were made available to users via www.soscisurvey.de.

\section{Analysis}

\section{Force Plate}

Raw data were visually inspected; artifacts and the first three and the last three seconds of every phase were removed. A low-pass filter (Butterworth 2nd order) was applied offline using Vicon Nexus 2.7.1 (Vicon Motion Systems Ltd. UK). We measured the root mean square (RMS), defined as the square root of the mean square power over a specified time interval (Merletti, 1999) over a $60 \mathrm{~s}$ period. This measure summarizes the variance in the participant's lateral position over time, relative to their average lateral position over the duration of the recording, with higher RMS values indicating greater movement. Further, we measured the mean power frequency (MPF), which calculates the average frequency within a power spectrum (Winter \& Patla, 1997). Higher MPF values indicating greater and faster movements. RMS and MPF were calculated in MATLAB. To analyze statistically the influence of the moving conditions on the body sway a t-test was performed comparing the mean medio-lateral movement in the two baselines (phase 1 and 2) with the medio-lateral movement in experimental phase for both conditions (social condition and non-social control), respectively. Resulting $p$-values were rectified using the Bonferroni adjusted alpha levels of $p=.0125$ per test $(p=.05 / 4)$.

\section{Questionnaire}

The ability to empathize with others was measured by the QCAE (Reniers et al., 2011), which differentiates between cognitive and affective empathy and consists of five principal factors (cognitive empathy: perspective taking and online simulation; affective empathy: emotion contagion, proximate responsivity and peripheral responsivity) and 31 items. The authors have verified its reliability (Cronbach's $\alpha$ between .70 and .85). For each participant, we calculated a mean score for "cognitive empathy" and for "affective empathy". The participant's current mood state was measured by the MDBF (Steyer et al., 1994), which consists of three scales: good and bad mood, alertness and fatigue, rest and restlessness, each consisting of 4 items, with an internal consistency of .92 (Cronbach's alpha) (Hinz et al., 2012). For each participant, we calculated a mean score for "mood". We used Pearson's correlation to investigate whether "cognitive empathy", "affective empathy", and "mood" scores had an influence on the degree of movement coordination during the test phase, by correlating the questionnaire scores with the delta values of the body sway (i.e., test phase minus the average of the two baselines; calculated separately for RMS and MPF). Resulting $p$-values were rectified using the Bonferroni adjusted alpha levels of $p=.0125$ per test (.05/4).

\section{Results}

The RMS of the medio-lateral movement differed significantly between experimental phase and the mean of the two baselines 
in the social condition with the avatar $(\mathrm{t}(42)=2.925, p=.006)$ : participants moved more in the experimental phase than in the baseline phases (experimental phase avatar (social condition) $\mathrm{M} \pm \mathrm{SD}: 2.24 \pm 3.33 \mathrm{~mm}$; baseline phase (1 and 2) $2.17 \pm$ $3.29 \mathrm{~mm}$ ). A post-hoc analysis with $\mathrm{G}^{*}$ power $^{2}$ (Faul et al., 2007) revealed a power (1- - of .9 and an effect size of dz. = .45. No significant difference was found for the RMS between the experimental phase of the non-social control condition and its baseline $(p>.0125$; experimental phase column (non-social control condition) $\mathrm{M} \pm \mathrm{SD}: 2.17 \pm 3.26 \mathrm{~mm}$; baseline phase (1 and 2) $2.16 \pm 3.35 \mathrm{~mm}$ ). No significant effects were found for the MPF data of the medio-lateral movement. There were no significant correlations between the questionnaire scores and the delta values of RMS and MPF in either the social condition or the non-social control condition (see Table 1).

\section{Discussion}

The aim of the current study was to design a human-avatar body sway synchronization paradigm modeled after real-life social coordination as a reliable setup for well-controlled synchrony studies. As a measure we used natural body sway, which is a naturalistic, yet accurately measurable expression of body movement. In line with our first two hypotheses, we found that the projection of a moving human-like avatar induced a corresponding greater movement in the participants, while no such effect was found for a moving neutral object (i.e., a same-sized column). This, firstly, demonstrates that the participant's movement was not simply caused by basal motion perception processes and secondly, proofs that our human-avatar body sway synchronization paradigm works in modeling interpersonal coordination and social synchrony effects without triggering this explicitly. The fact that there was an increase of RMS values only in the social condition with the avatar shows that people moved laterally beyond their natural body sway only when being exposed to a human-like avatar, but not when perceiving a neutral object. These findings are in line with a previous study that presented participants with point-light displays of a balancing gymnast (Tia et al., 2011): participants' body sway only increased when observing an upright representation of the gymnast, but not when the point-lights were displayed upside down. Consequently, the authors argued that the optical flow of the moving point-lights alone was not sufficient to increase body sway, when they did not represent a human figure.

The treatment in our study, however, did not influence the participants' body sway frequency in a systematic way. The analysis of the MPF values showed that the participants did

\footnotetext{
${ }^{2}$ Due to a lack of comparable studies, we could not estimate a likely effect size for our current study. Therefore, no a-priori power analysis was conducted and sample size was determined via Mead's resource equation (Mead, 1990).
}

Table 1 Correlations (Pearson) of the questionnaire ratings, cognitive empathy (CE), affective empathy (AE), and mood with the delta values (RMS and MPF) of the social condition and non-social control

\begin{tabular}{lllllll}
\hline & & \multicolumn{2}{l}{ Social Condition } & & \multicolumn{2}{l}{ Non-Social Control } \\
\cline { 3 - 4 } \cline { 6 - 7 } \cline { 6 - 7 } & & RMS & MPF & & RMS & MPF \\
\hline \multirow{2}{*}{ CE (mean) } & $\mathrm{r}_{\mathrm{s}}$ & .233 & .243 & & -.007 & .327 \\
& $\mathrm{p}$ & .13 & .13 & & .97 & .04 \\
AE (mean) & $\mathrm{r}_{\mathrm{s}}$ & .021 & .157 & & .263 & .168 \\
& $\mathrm{p}$ & .90 & .33 & & .09 & .29 \\
Mood (mean) & $\mathrm{r}_{\mathrm{s}}$ & .132 & .131 & & -.232 & .151 \\
& $\mathrm{p}$ & .40 & .42 & & .13 & .34 \\
\hline
\end{tabular}

resulting p-values were rectified using Bonferroni adjusted alpha levels of $\mathrm{p}=.0125$ per test $(.05 / 4)$.

not consistently change their swaying frequency, neither when being exposed to a human-like avatar nor when perceiving the neutral object. This lack of an effect might have been caused by the fact that the frequency of avatar's and the column's movement and the frequency of the participants' natural body sway were already very similar to one another, as we specifically chose a swaying frequency that approximated natural body sway. Therefore, it would be important for future studies to investigate whether different swaying frequencies of the avatar are actually able to alter a participant's swaying frequency.

Contrary to our third hypothesis, the ability to empathize with others and the degree of positive mood, assessed by selfevaluation, were not correlated with a stronger change of body sway in response to the moving projected image in either the social condition with the avatar or the non-social control. According to previous studies, we would have expected participants that scored higher on empathic abilities and those with a better mood to respond more to the swaying avatar (Chartrand \& Bargh, 1999; Sonnby-Borgström et al., 2003; Van Baaren et al., 2006). However, previous findings on the connection between interpersonal coordination and dispositional empathy and mood are not completely consistent even for human-human interactions. While Chartrand and Bargh (1999) found only a positive effect of participants' perspective taking abilities, but not of their empathic concern on behavior mimicry, Sonnby-Borgström et al. (2003) demonstrated that emotional, rather than cognitive aspects of participants' empathy predicted increased facial mimicking. although, some studies find that participants in a good mood mimic a confederate significantly more often than participants in a negative mood (e.g. Van Baaren et al., 2006), a recent study on interpersonal synchrony did not find any evidence that positive mood induced through videos or affective picture sets promoted interpersonal synchrony (Fujiwara \& Daibo, 2018). Nonetheless, it is also possible that our negative results arose from the fact that we specifically chose a rather neutrallooking avatar. Especially empathic abilities, which are 
enhanced through a feeling of similarity (Valdesolo \& DeSteno, 2011), might influence coordination with a real person more strongly than coordination with an avatar. Overall, our results show that the avatar's movement triggered the same degree of body sway, irrespective of the participant's empathic abilities and mood state. Further studies investigating the effect of empathy and mood state on human-avatar coordination would be needed.

\section{Conclusion}

Previous research demonstrated that interpersonal coordination is greatly influenced by characteristics of the interaction partners, like the cultural homogeneity of a group (Néda et al., 2000), shared goals (Reddish et al., 2013), and the likability of the other person (Miles et al., 2010). Considering that most research questions necessitate an experimental set-up without such uncontrolled biases inherent to the interactions between real persons, our human-avatar body sway synchronization paradigm provides a promising avenue in the direction of controlled experimental studies to investigate possible causations on interpersonal coordination. It thus, builds a basic fundament to reduce the uncontrolled influence of pre-existing rapport on interpersonal coordination.

\section{Limitations}

The study is limited by the fact more women (35) than men (14) participated in the experiment and further by a small sample size, however a post-hoc analysis revealed a power of .9 and an effect size of dz. $=.45$.

Authors' Contributions All authors [Petra Jansen, Lisa Horn and Clara Scheer] contributed to the conception and design of the experiment. Material preparation, data collection and analysis were performed by [Clara Scheer]. The first draft of the manuscript was written by [Clara Scheer] and all authors commented on previous versions of the manuscript. All authors read and approved the final manuscript.

Funding (Information that Explains whether and by Whom the Research Was Supported) Open Access funding enabled and organized by Projekt DEAL. Not applicable - This research did not receive any specific grant from public, commercial or non-profit funding agencies.

Availability of Data and Material (Data Transparency) All data are available if needed by the reviewers or the journal.

Code Availability (Software Application or Custom Code) Not applicable.

\section{Declarations}

Conflicts of Interest/Competing Interests (Include Appropriate Disclosures) Not applicable - The authors declare that they have no conflicts of interest to disclose.
Ethics Approval (Include Appropriate Approvals or Waivers) This study was performed in line with the principles of the Declaration of Helsinki. Approval was granted by the Ethics Committee of the University of Regensburg (Date 11-21-2018/No. 18-1202-101).

Consent to Participate (Include Appropriate Statements) All participants included in the study filled out and signed written informed consent to participate in the experiment.

Consent for Publication (Include Appropriate Statements) Participants were informed about and agreed on the publication of the data. All data are anonymized.

Open Access This article is licensed under a Creative Commons Attribution 4.0 International License, which permits use, sharing, adaptation, distribution and reproduction in any medium or format, as long as you give appropriate credit to the original author(s) and the source, provide a link to the Creative Commons licence, and indicate if changes were made. The images or other third party material in this article are included in the article's Creative Commons licence, unless indicated otherwise in a credit line to the material. If material is not included in the article's Creative Commons licence and your intended use is not permitted by statutory regulation or exceeds the permitted use, you will need to obtain permission directly from the copyright holder. To view a copy of this licence, visit http://creativecommons.org/licenses/by/4.0/.

\section{References}

Bernieri, F. J., \& Rosenthal, R. (1991). Interpersonal coordination: Behavior matching and interactional synchrony. In R. S. Feldman \& B. Rime (Eds.), Fundamentals of nonverbal behavior (pp. 401432). Cambridge University Press.

Bernieri, F., Reznick, J. S., \& Rosenthal, R. (1988). Synchrony, Pseudosynchrony, and Dissynchrony: Measuring the entrainment process in mother-infant interactions. Article in Journal of Personality and Social Psychology, 54(2), 243-253. https://doi. org/10.1037/0022-3514.54.2.243.

Chang, A., Kragness, H. E., Livingstone, S. R., Bosnyak, D. J., \& Trainor, L. J. (2019). Body sway reflects joint emotional expression in music ensemble performance. Scientific Reports, 9(1), 1-11. https://doi.org/10.1038/s41598-018-36358-4.

Chang, A., Kragness, H, E., Tsou, W., Bosnyak, D, J., Thiede, A., \& Trainor, L, J. (2020). Body sway predicts romantic interest in speed dating. Social Cognitive and Affective Neuroscience, June, 1-8. https://doi.org/10.1093/scan/nsaa093

Chartrand, T. L., \& Bargh, J. A. (1999). The Cameleon effect: The perception-behavior LInk and social interaction. Journal of Personality and Social Psychology, 76(6), 893-910. https://doi. org/10.1037/0022-3514.76.6.893.

Chartrand, T. L., \& Lakin, J. L. (2013). The antecedents and consequences of human behavioral mimicry. Annual Review of Psychology, 64(1), 285308. https://doi.org/10.1146/annurev-psych-113011-143754.

Chartrand, T. L., \& van Baaren, R. B. (2009). Human Mimikry. In M. P. Zanna (Ed.), Advances in Experimental Social Psychologyp (1st ed., Vol. 41, Issue 2, pp. 219-274). Academic Press.

Chartrand, T. L., Maddux, W. W., \& Lakin, J. L. (2005). Beyond the perception-behavior link: The ubiquitous utility and motivational moderators of nonconscious mimicry. In R. R. Hassin, J. S. Uleman, \& J. A. Bargh (Eds.), The new unconscious (pp. 334-361). Oxford Univ. Press.

Condon, W. S., \& Sander, L. W. (1974). Synchrony demonstrated between movements of the neonate and adult speech. Child Development, 45(2), 456-462. https://doi.org/10.2307/1127968. 
de Bruin, G., Ypey, D. L., \& Van Meerwijk, W. P. M. (1983). Synchronization in chains of pacemaker cells by phase resetting action potential effects. Biological Cybernetics, 48(3), 175-186. https://doi.org/10.1007/BF00318085.

Faul, F., Erdfelder, E., Lang, A. G., \& Buchner, A. (2007). G*power 3: A flexible statistical power analysis program for the social, behavioral, and biomedical sciences. Behavior Research Methods, 39(2), 175191. https://doi.org/10.3758/BF03193146.

Fujiwara, K., \& Daibo, I. (2018). Affect as an antecedent of synchrony: A spectrum analysis with wavelet transform. Quarterly Journal of Experimental Psychology, 71(12), 2520-2530. https://doi.org/10. $1177 / 1747021817745861$

Funato, T., Aoi, S., Tomita, N., \& Tsuchiya, K. (2016). Smooth enlargement of human standing sway by instability due to weak reaction floor and noise. Royal Society Open Science, 3(1). https://doi.org/10. 1098/rsos. 150570 .

Guéguen, N., \& Martin, A. (2009). Incidental similarity facilitates behavioral mimicry. Social Psychology, 40(2), 88-92. https://doi.org/10. 1027/1864-9335.40.2.88

Hagen, E, H., \& Bryant, G, A. (2003). Music and dance as a coalition signaling system. In Human Nature. https://doi.org/10.1007/ s12110-003-1015-Z

Haken, H., Kelso, J. A. S., \& Bunz, H. (1985). A theoretical model of phase transitions in human hand movements. Biological Cybernetics, 51(5), 347-356. https://doi.org/10.1007/BF00336922.

Hinz, A., Daig, I., Petrowski, K., \& Brähler, E. (2012). Die Stimmung in der deutschen Bevölkerung: Referenzwerte für den Mehrdimensionalen Befindlichkeitsfragebogen MDBF. PPmP Psychotherapie · Psychosomatik - Medizinische Psychologie, 62(02), 52-57. https://doi.org/10.1055/s-0031-1297960.

Lakin, J. L., \& Chartrand, T. L. (2003). Using nonconscious behavioral mimicry to create affiliation and rapport. Psychological Science, 14(4), 334-339. https://doi.org/10.1111/1467-9280.14481.

Lang, P., Bradley, M., \& Cuthbert, B. (2008). International affective picture system (IAPS): Affective ratings of pictures and instruction manual. Technical report A-8 University of Florida.

Leiner, D. J. (2019). SoSci Survey (3.1.06). https://www.soscisurvey.de. Lumsden, J., Miles, L. K., Richardson, M. J., Smith, C. A., \& Macrae, C. N. (2012). Who syncs? Social motives and interpersonal coordination. Journal of Experimental Social Psychology, 48(3), 746-751. https://doi.org/10.1016/j.jesp.2011.12.007.

McIntosh, D. N. (2006). Sponteous facial mimicry, liking and emotional contagion. Polish Psychological Bulletin, 37(1), 31-42.

Mead, R. (1990). The design of experiments : Statistical principles for practical applications. Cambridge University Press.

Meerhoff, L. R. A., de Poel, H. J., Jowett, T. W. D., \& Button, C. (2017). Influence of gait mode and body orientation on following a walking avatar. Human Movement Science, 54(June), 377-387. https://doi. org/10.1016/j.humov.2017.06.005.

Merletti, R. (1999). Standards for reporting EMG data*. Journal of Electromyography and Kinesiology, 243-246. https://doi.org/10. 1201/9781420036985.ax2.

Miles, L. K., Griffiths, J. L., Richardson, M. J., \& Neil Macrae, C. (2010). Too late to coordinate: Contextual influences on behavioral synchrony. European Journal of Social Psychology, 40, 52-60. https://doi.org/10.1002/ejsp.

Mondillon, L., Niedenthal, P. M., Gil, S., \& Droit-Volet, S. (2007). Imitation of in-group versus out-group members' facial expressions of anger: A test with a time perception task. Social Neuroscience, 2(3-4), 223-237. https://doi.org/10.1080/17470910701376894.

Néda, Z., Ravasz, E., Brechet, Y., Vicsek, T., \& Barabási, A. L. (2000). Self-organization in the concert hall: The dynamics of rhythmic applause. Nature, 403(6772), 849-850. https://doi.org/10.1038/ 35002660 .
Nishiyama, M., Miyauchi, T., Yoshimura, H., \& Iwai, Y. (2016). Synthesizing realistic image-based avatars by body sway analysis. HAI 2016 - proceedings of the 4th international conference on human agent interaction, 155-162. https://doi.org/10.1145/2974804.2974807.

Ravignani, A. (2015). Evolving perceptual biases for antisynchrony: A form of temporal coordination beyond synchrony. Frontiers in Neuroscience, 9(SEP), 1-6. https://doi.org/10.3389/fnins.2015.00339.

Reddish, P., Fischer, R., \& Bulbulia, J. (2013). Let's dance together: Synchrony, shared intentionality and cooperation. PLoS One, 8(8), e71182. https://doi.org/10.1371/journal.pone.0071182.

Reniers, R. L. E. P., Corcoran, R., Drake, R., Shryane, N. M., \& Völlm, B. A. (2011). The QCAE: A questionnaire of cognitive and affective empathy. Journal of Personality Assessment, 93(1), 84-95. https:// doi.org/10.1080/00223891.2010.528484.

Reynolds, R. F., \& Osler, C. J. (2014). Mechanisms of interpersonal sway synchrony and stability. Journal of the Royal Society Interface, 11(101), 20140751. https://doi.org/10.1098/rsif.2014.0751.

Richardson, M. J., Marsh, K. L., Isenhower, R. W., Goodman, J. R. L., \& Schmidt, R. C. (2007). Rocking together: Dynamics of intentional and unintentional interpersonal coordination. Human Movement Science, 26(6), 867-891. https://doi.org/10.1016/j.humov.2007.07.002.

Sacheli, L. M., Candidi, M., Era, V., \& Aglioti, S. M. (2015). Causative role of left aIPS in coding shared goals during human-avatar complementary joint actions. Nature Communications, 6(May), 1-11. https://doi.org/10.1038/ncomms8544.

Schmidt, R. C., \& Richardson, M. J. (2008). Dynamics of interpersonal coordination. In A. Fuchs \& V. K. Jirsa (Eds.), Coordination: Neural, behavioral and social dynamics. Understanding Complex Systems. Springer. https://doi.org/10.1007/978-3-540-74479-5_14.

Sonnby-Borgström, M., Jönsson, P., \& Svensson, O. (2003). Emotional empathy as related to mimicry reactions at different levels of information processing. Journal of Nonverbal Behavior, 27(1), 3-23. https://doi.org/10.1023/A:1023608506243.

Steyer, R., Schwenkmezger, P., Notz, P., \& Eid, M. (1994). Testtheoretische Analysen des Mehrdimensionalen Befindlichkeitsfragebogen (MDBF) [theoretical analysis of a multidimensional mood questionnaire (MDBF)]. Diagnostica, 40(4), 320-328 https://psycnet.apa.org/record/1995-86418-001.

Tia, B., Saimpont, A., Paizis, C., Mourey, F., Fadiga, L., \& Pozzo, T. (2011) Does observation of postural imbalance induce a postural reaction? PLoS One, 6(3), e17799. https://doi.org/10.1371/journal.pone.0017799.

Valdesolo, P., \& DeSteno, D. (2011). Synchrony and the social tuning of compassion. Emotion, 11(2), 262-266. https://doi.org/10.1037/ a0021302.

Van Baaren, R. B., Fockenberg, D. A., Holland, R. W., Janssen, L., \& Van Knippenberg, A. (2006). The moody chameleon: The effect of mood on non-conscious mimikry. Social Cognition, 24(4), 426-437.

Van Ulzen, N., Lamoth, C., Daffertshofer, A., \& Semin, G, R. (2007). Characteristics of instructed and uninstructed interpersonal coordination while walking side-by-side. Neuroscience Letters, 1-6. https://doi.org/10.1016/j.neulet.2007.11.070.

Vicaria, I. M., \& Dickens, L. (2016). Meta-analyses of the intra- and interpersonal outcomes of interpersonal coordination. Journal of Nonverbal Behavior, 40(4), 335-361. https://doi.org/10.1007/ s10919-016-0238-8

Wiltermuth, S. S., \& Heath, C. (2009). Synchrony and cooperation. Psychological Science, 20(1), 1-5. https://doi.org/10.1111/j.14679280.2008.02253.x.

Winter, D., \& Patla, A. (1997). Signal processing and linear systems for the movement sciences. Waterloo: Waterloo Biomechanics.

Publisher's Note Springer Nature remains neutral with regard to jurisdictional claims in published maps and institutional affiliations. 